\title{
FULMINATING LYMPHOGRANULOMA VENERUM IN AN HIV-POSITIVE MIDDLE AGED PATENT MASCURADING AS SOFT TISSUE SARCOMA.
}

\author{
1*Adigan, Imanila Abiena, ${ }^{2}$ Padcyi, Abayomi aud ${ }^{2}$ Adcgboro, Boxz \\ 'Division of Plostic and Reconstructive Surgery, Department of Surgery, ${ }^{2}$ Department of Medical Microbiology, \\ University of llorin Teaching Fowpital, llorin, Nigeria.
}

* Correspondence author e-mail:

Tel: 08033464868,08051750085

\begin{abstract} positive results.

KEY WORDS: LGV, HIV, Sarcoma

\section{INTRODUCTION}

Lymphogranuloma venerum (LGV) is a systemic sexually transmitted disease (STD) caused by Chlamydia trachomatis serotypes L1, L2 and L3. LGV is endemic in parts of Africa, Asia, South America and the Caribbean but rare in Western countries where the disease occurs mainly in sporadic form (1).
\end{abstract}

This is a case report of a 40-year old male patient presenting with a 17-month history of right originalgrowing swellings. Initial chemical assessment was suggestive of soft tissue sarcoma, and he was admitted in the surgical ward. Histopathology investigation suggested inflammatory process was taking place. Assessment for Chlarydia IgG with Enzyme-Linked Immunosorhent Assay (ELISA) using Immumocomb (Organics, Israel) gave a positive titre of 1 in 32. HIV serology using p24 ELISA and Western Blotting gave

He made significant progress after two woeks on antibioties and antiretroviral (HAART) therapy.

Large outbreaks of LGV occurred recently in Europe and America mostly among men who have sex with men (MSM) $(2,3,4)$. Most of the infected men were co-infected with human immumodeficiency virus (HIV) and had atypical presentation. HIV infected persons with LGV have more extensive disease, and this may cause diagnostic confusion as found in this patient being reported. We present here therefore a case of fulminating LGV in a middle aged man with HIV infection who presented with signs and symptoms suggestive of sof tissue sarcoma (STS) of the thigh.

\section{CASE PRESNTATION}

Mr. B is a 40-year-old Nigerian who had tertiary education and resident in Lagos. He has history of 17 month right inguinal/groin ulcerating swelling, 12-month right leg multiple ulcerating swellings and 3-month progressive weight loss. Lesions had started as fluctuant swellings of the affected areas with some treatment received before presentation at the University of Morin Teaching Hospital including incision and drainage on right inguinal and leg swellings. This resulted in progressive increase in wound size, failure of wound to heal and production of sero-purulent discharge. No history suggestive of primary LGV. No identifiable risk factor. The only admitted sexual partner (wife) was apparently healthy at the time the patient presented. On examination, there was lymphoedema onightlower limb, right inguinal groin ulcerating mass which together measures $20 \mathrm{~cm} \times 18 \mathrm{~cm}$ (Figures 1 ) with raised irregular edges and right leg, below the knee joint ulcer measuring $10 \mathrm{~cm} \times 12 \mathrm{~cm}$ with sloppy odge. The 
ulcers were all producing offensive seropurulent discharge. The anterior abdominal wall veins were distended; the patient was chronically wasted and had left inguinal lymphadenopathy. A clinical assessment of soft tissue sarcoma (STS) was made and patient admitted for invertigations. Significant results included that of histopathology that was not suggestive of malignancy but revealed focus of inflammation and serological assessment for Chlamydia IgG with ImmumComb (Orgenics, Israel) that gave significant titre (1:32). Patient was confirmod HIV

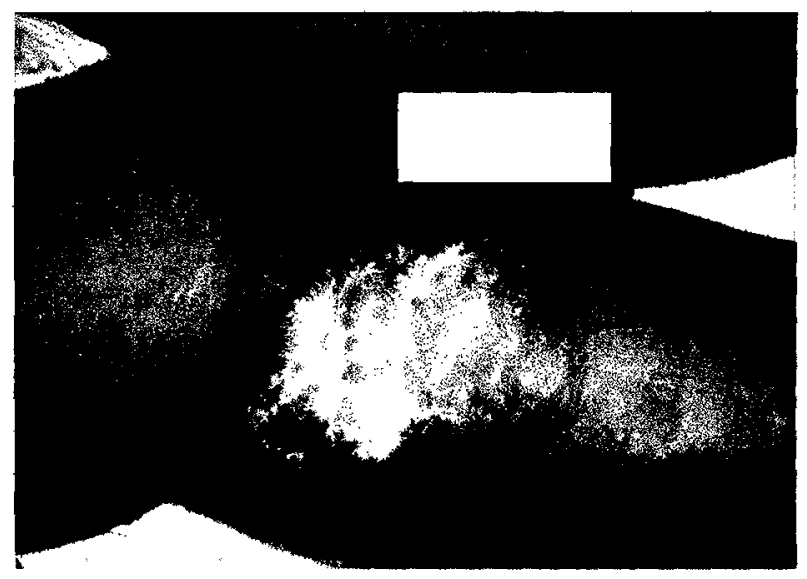

Figure 1: Fumigating left groin / inguinal mass before treatment

\section{DISCUSSTON}

The clinical course of LGV can be divided into the primary, secondary and the late stages. The prymary lesions may not be noticed by infected people (1) as in this patient. He presented eartier to a medical practitioner, during the secondary stage, who aspirated the swellings before doing incision and drainage that worsened the patient's condition. On presentation in our hospital, diagnosis of LGV was not strongly considered until histological assessment of the masses was done. Reason being that rarely do we see LGV in our hospital in recent time despite the claim that positive by p24 EIISA and Westem Blotting techniques. Treatment given included doxycycline $100 \mathrm{mg}$ bd for more than 21 days, twice daily drescing of wounds with honey and highly active anti-retroviral therapy (HAART) including Starvudine-400mg b.d, Nevirapine-200mg b.d and Lamivudine. $150 \mathrm{mg}$ b.d. Patient improved significantly as evidenced by the wounds reducing size, less discharging and subsiding lymphoedema (Figure 2).

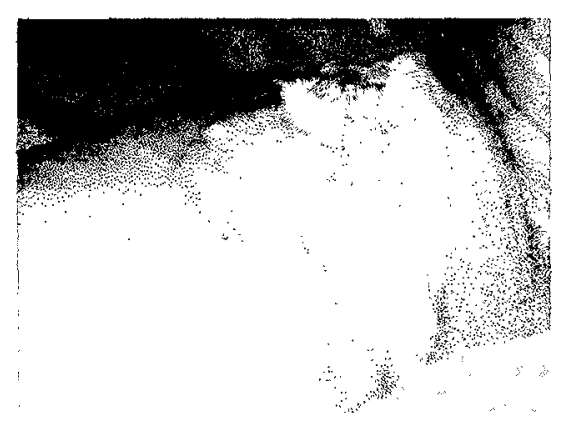

Figure 2: Funigating left groin/ inguinal mass after two weeks treatment

the disease is endemic in Africa. Besides, the association of LGV with HIV caused the deviation from the classical presentation pattern. This however is in line with resent reponts from the Westem industrialized nations $(2,3,4)$ although the patient denied history of homosexualism and did not have features of colitis or procto-colitis. The only admitted sexual partner (wife) was apparently healthy at the time of presentation. What then are the other factors at play?

There were diagnostic challenges. The patient wife who was clinically healthy could not be 
screened for HIV and Chlamydia infection due to socio-cultural barriers. The serological kit used to screen for Chlamydia, though certified all right by the manufacturer, would have been more useful if IgM rather than IgG was assayed for. Diagnostic challenges not withstanding, the patient responded well to the medications given. We recommend that medical practitioners should not forget to exclude LGV before incising any inguinal fluctuant swellings and screen for HIV in suspected LGV cases.

\section{REFERENCES}

1. Robert BJ. LGV In: Mandel, G.L et al (eds), Principles \& Practice of infectious diseases (4th Edition) CLS. 1995: 1679. 1693.

2. Kropp RY, Wong T. Emergence of lymphogranuloma venerum in Canada. Can. Med. Assoc. J.2005; 172 (13): 1674-1676.

3. Centers for Disease Control and Prevention. Lymphogranuloma venerum among men who have sex with menNetherlands, 2003-2004. MMMR 2004; 53 (42): 985-988.

4. Health Protection Agency. Initial results of enhanced surveillance for lymphogranuloma venerum in England. CDR Weekly 2005: 15 (4). 


\section{CORRECTIONS}

1. In our journal Vol8(2):107-113 we published an article titled "Intestinal Helminthiasis in Children in a subutb of Lagos, Nigeria:

A)

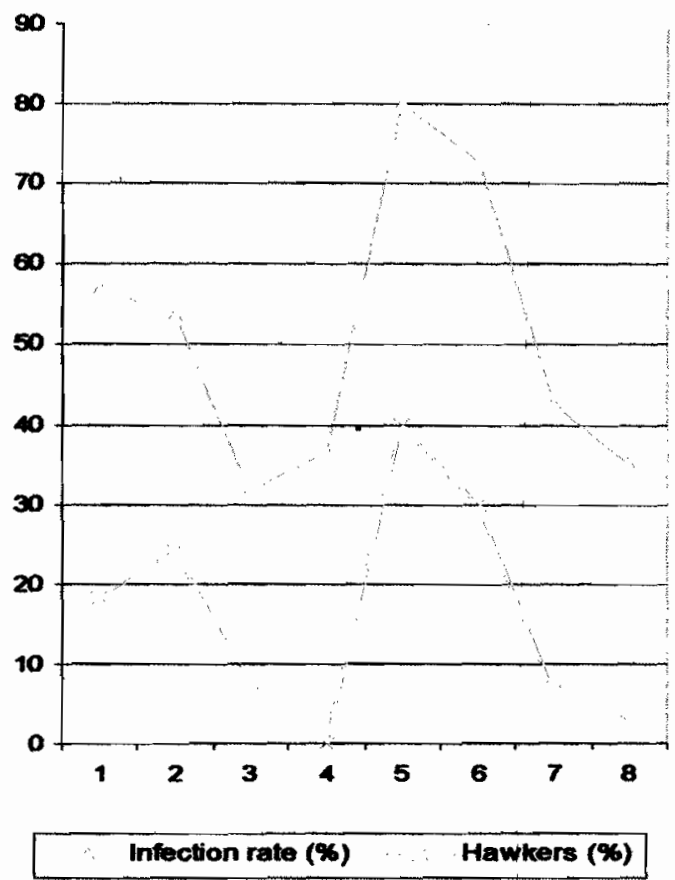

Evaluation of risk factons and Habits". We inadvertently omittod Table $1 \mathrm{c}$ in page 111 . This table is published below.
E)

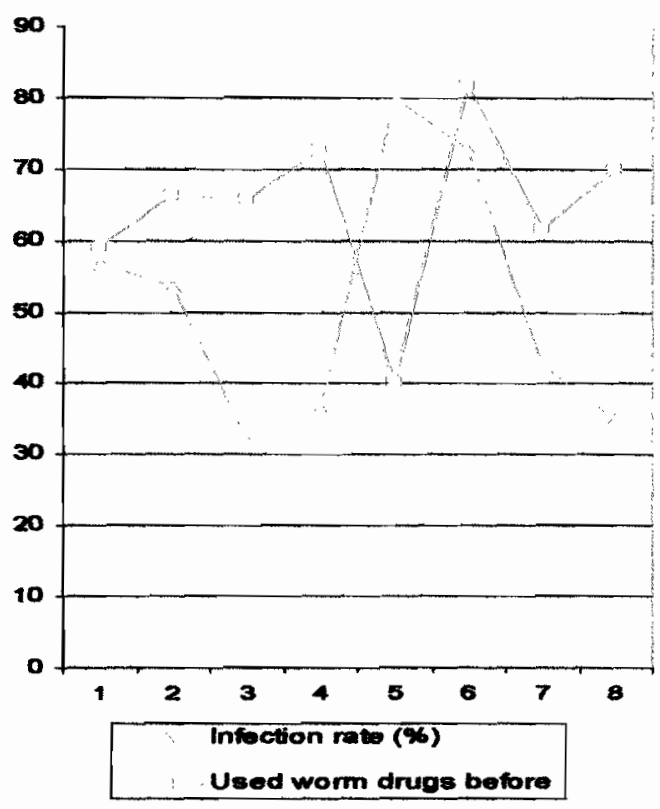

c)

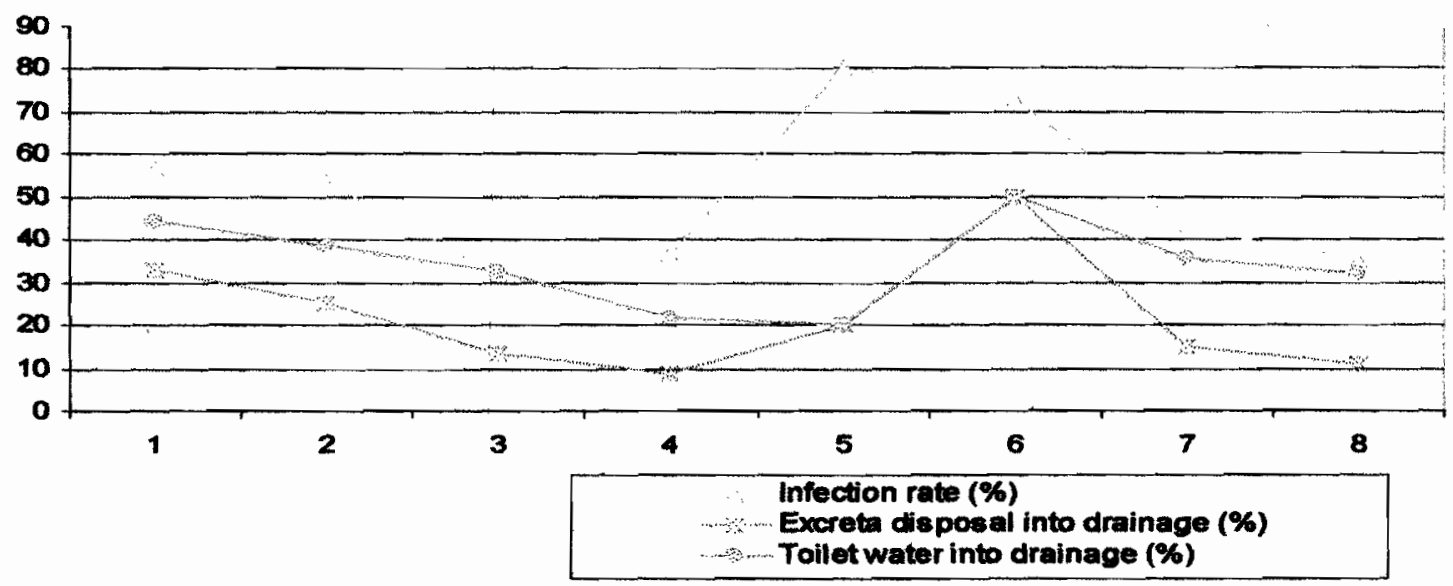

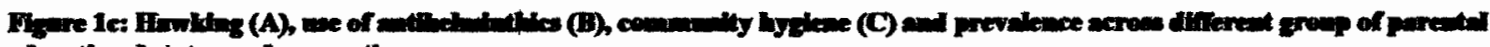

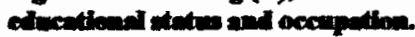

$Y$-exis = Positive values (\%); $X$-axis = Groups:

1 = Trading Fathers

4 = Professional Mothers

7 Educated Fathers

2 = Trating Mothers

$5=$ Uneduatod Fethers

$8=$ Educated Mothers

3 = Profesiososal Fathers

6 = Uneductted Mothers 
2. In our publication of May 2006 we gave

Omotosho's initials as J.O. instead of

J.A. It is corrected below.
Buhari, M. O. and Omotayo, J. A.; Viruses and Cancer - An Overview. Afr. J. Clin. Exp. Microbiol (2006) 7(2): 125-131 